-Supporting Information-

\title{
Ligands Affect Hydrogen Absorption and Desorption by Palladium Nanoparticles
}

\author{
Noah J. Johnson, ${ }^{1}$ Brian Lam, ${ }^{1}$ Rebecca S. Sherbo, ${ }^{1}$ David K. Fork, ${ }^{2}$ Curtis P. Berlinguette ${ }^{1,3,4,5 *}$ \\ ${ }^{1}$ Department of Chemistry, The University of British Columbia, 2036 Main Mall, Vancouver, \\ British Columbia, V6T 1Z1, Canada. \\ ${ }^{2}$ Google LLC, 1600 Amphitheatre Parkway, Mountain View, California 94043, USA. \\ ${ }^{3}$ Department of Chemical and Biological Engineering, The University of British Columbia, 2360 \\ East Mall, Vancouver, British Columbia, V6T 1Z3, Canada. \\ ${ }^{4}$ Stewart Blusson Quantum Matter Institute, The University of British Columbia, 2355 East \\ Mall, Vancouver, British Columbia, V6T 1Z4, Canada. \\ ${ }^{5}$ Canadian Institute for Advanced Research (CIFAR), 661 University Avenue, Toronto, Ontario, \\ M5G 1M1, Canada.
}

*Corresponding author. E-mail: cberling@chem.ubc.ca 

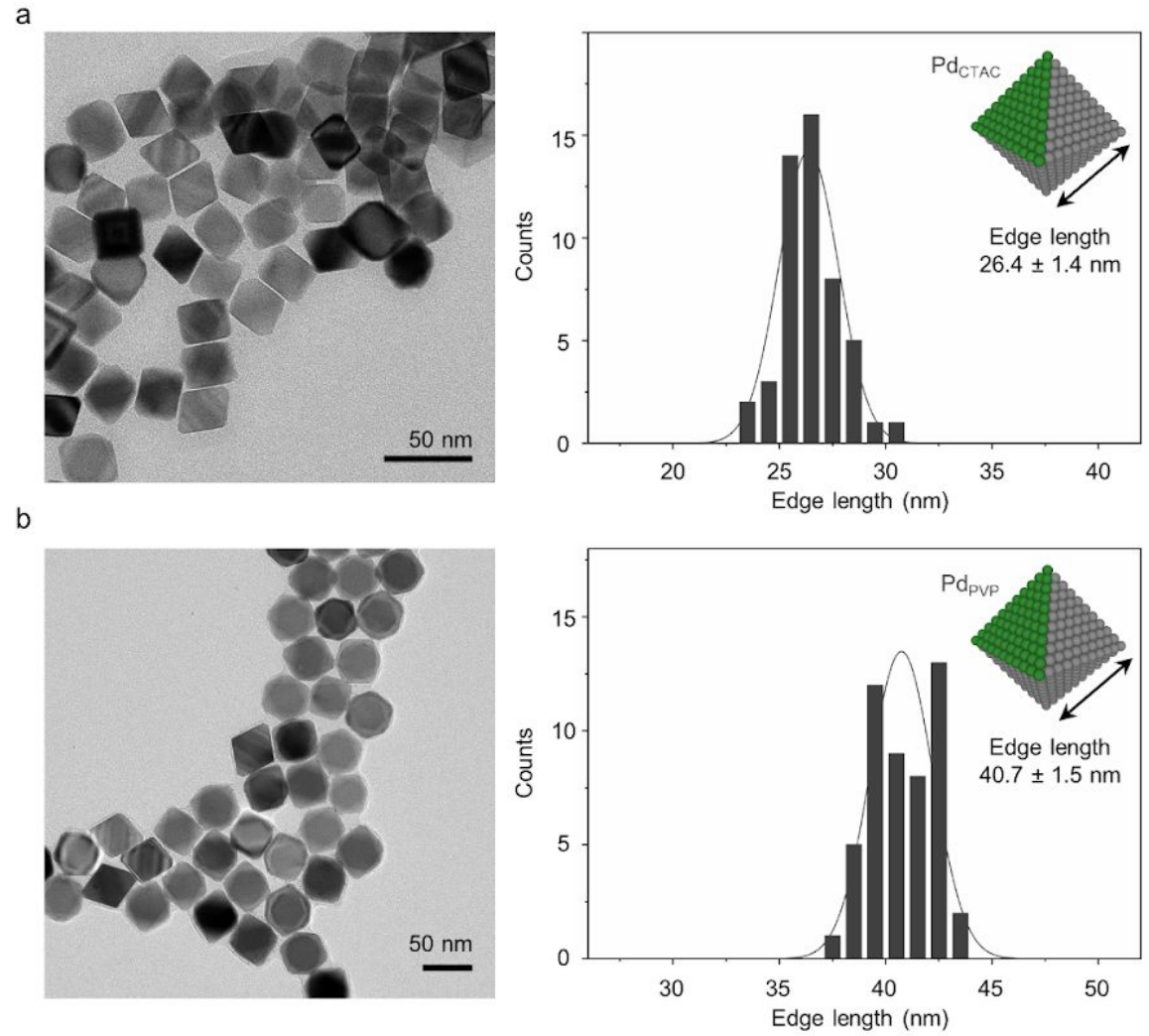

Figure S1. TEM image and corresponding size analysis of (a) $\mathbf{P d} \mathbf{C}_{\text {CTAC }}$ and (b) $\mathbf{P d}_{\mathbf{P V P}}$ octahedral nanoparticles respectively. Size analyses were obtained from about 50 randomly selected nanoparticles and manually measured using the measurement tool in Adobe Acrobat. 


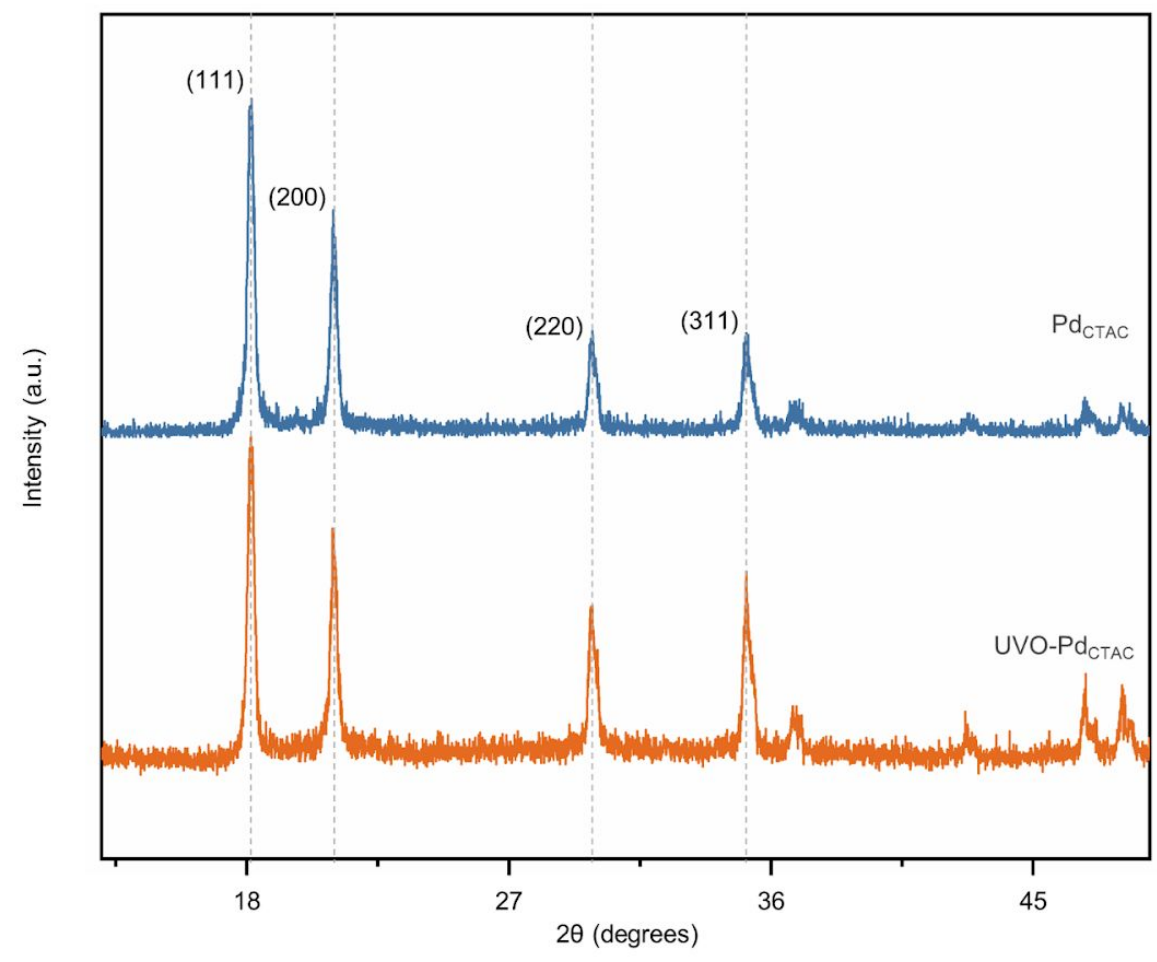

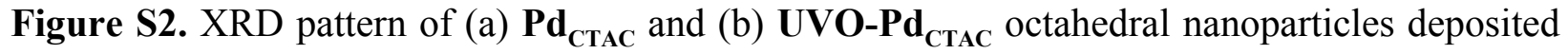
on a zero background sample holder at ambient temperature and pressure. Dotted vertical lines correspond to standard palladium reference pattern (JCPDS: 00-046-043). (X-ray source $=$ Mo; $\lambda$ $=0.7107 \AA$..) 


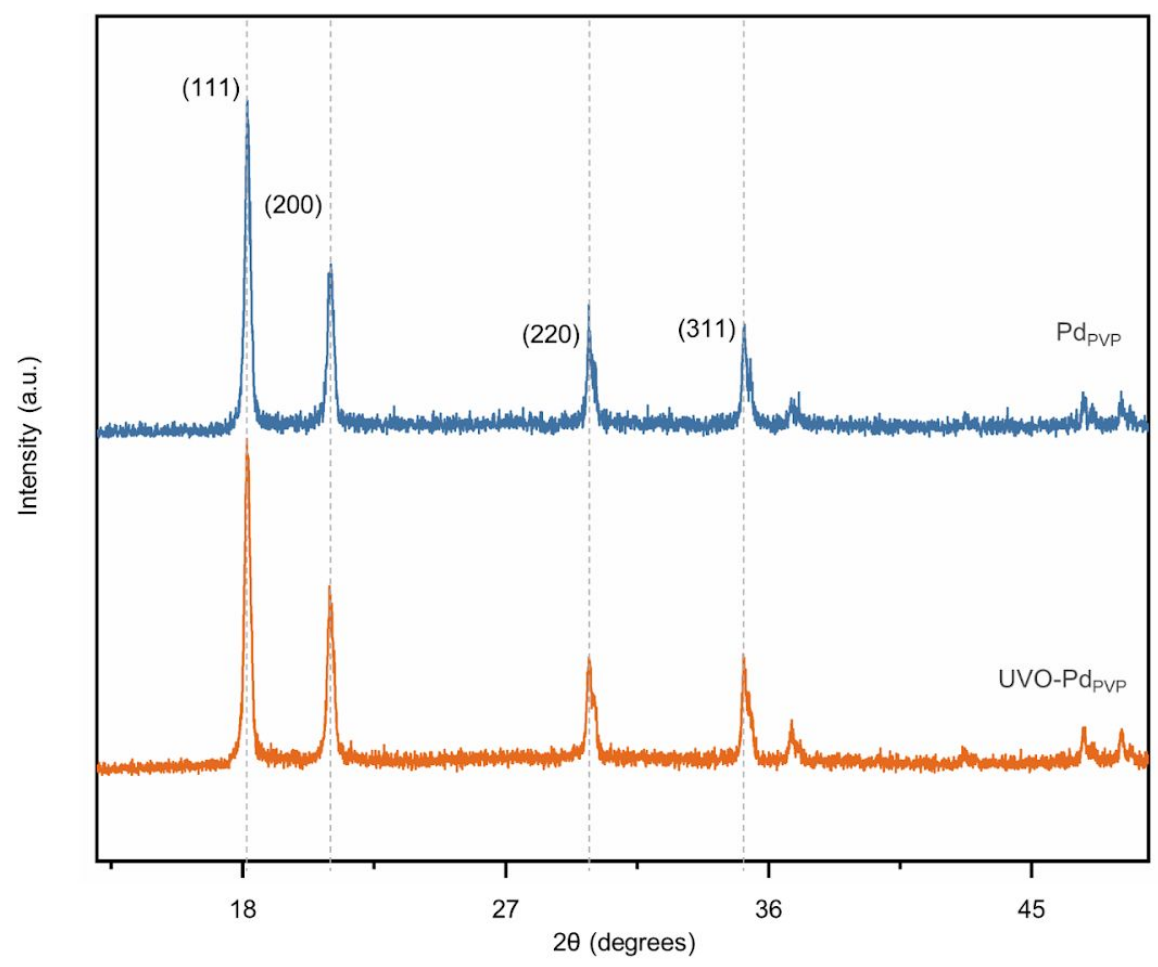

Figure S3. XRD pattern of (a) $\mathbf{P} \mathbf{d}_{\mathbf{P V P}}$ and (b) UVO-Pd $\mathbf{P V P}_{\mathbf{P}}$ octahedral nanoparticles deposited on a zero background sample holder at ambient temperature and pressure. Dotted vertical lines correspond to standard palladium reference pattern (JCPDS: 00-046-043). (X-ray source $=$ Mo; $\lambda$ $=0.7107 \AA$ А.) 
a

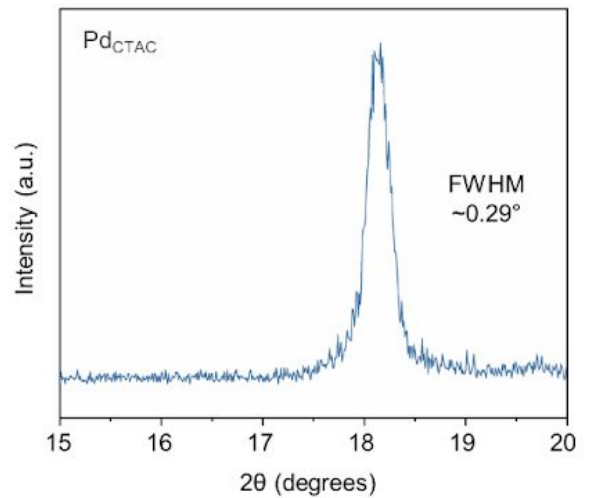

b

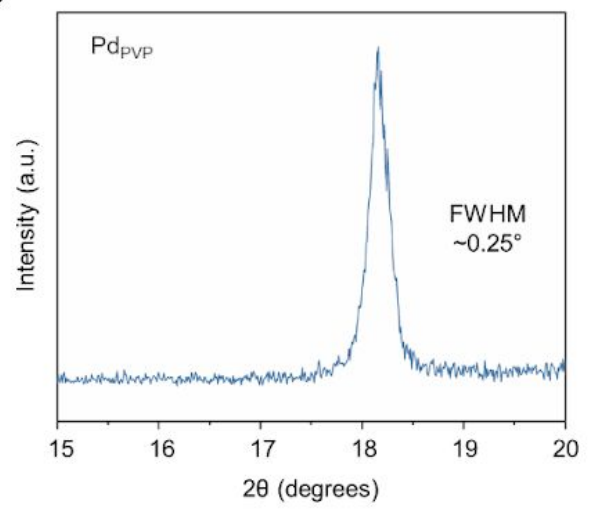

UVO-treated
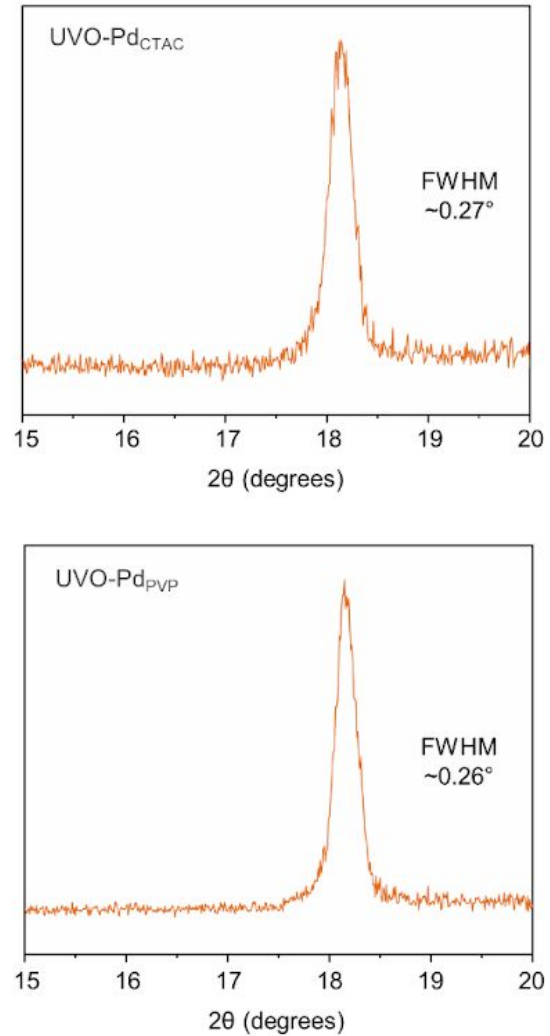

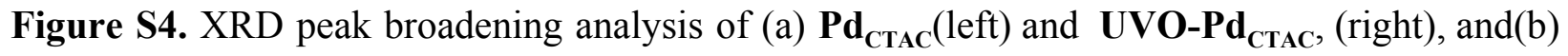

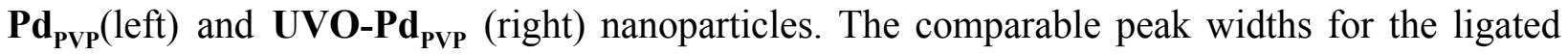
and UVO-treated nanoparticles confirm that the nanoparticle crystallite sizes are preserved during UVO treatment. (X-ray source $=$ Mo; $\lambda=0.7107 \AA$.) 

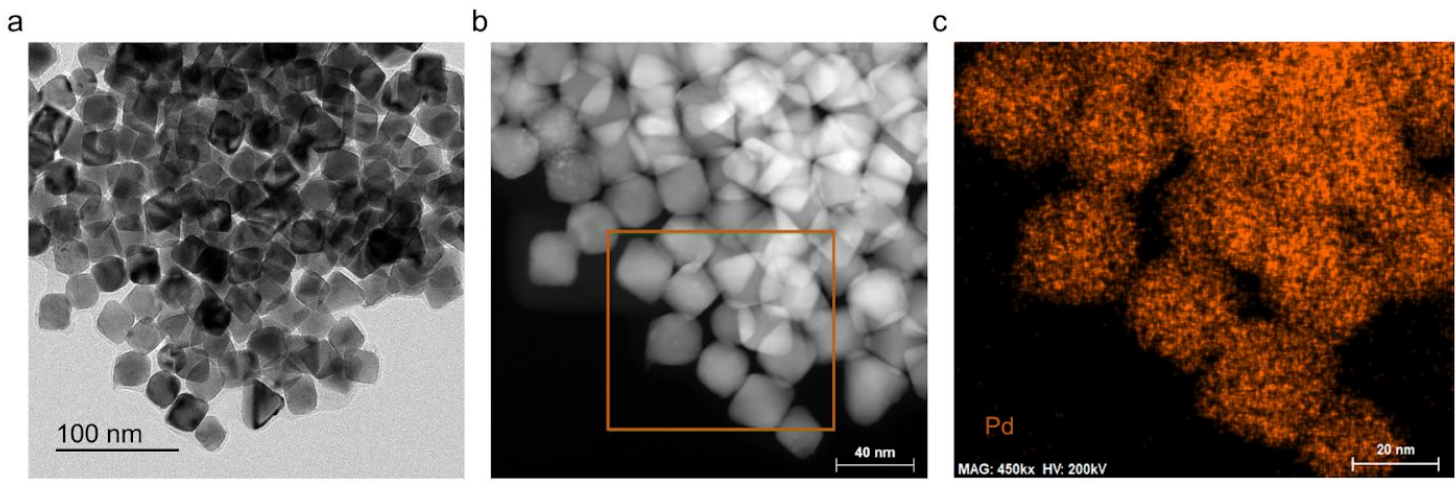

Figure S5. Microscopy analysis of UVO-Pd ${ }_{\text {CTAC }}$ nanoparticles, (a)TEM, (b) STEM, and (c) selected area elemental mapping by EDX. The images confirm the nanoparticles maintain their shape, size and composition after UVO treatment. 
a

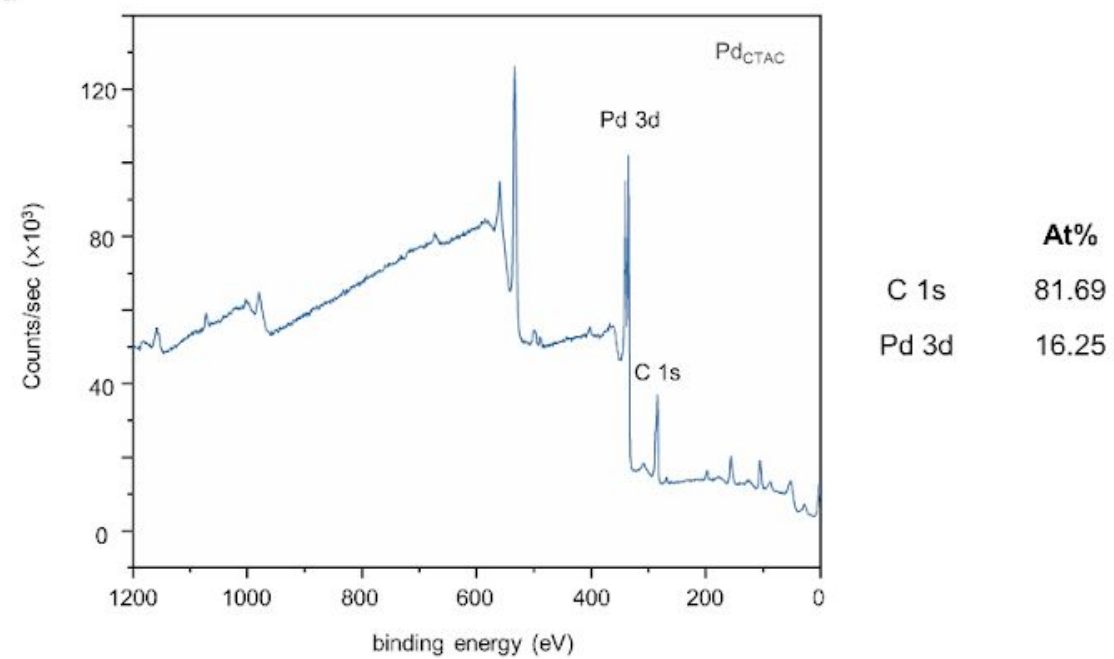

b

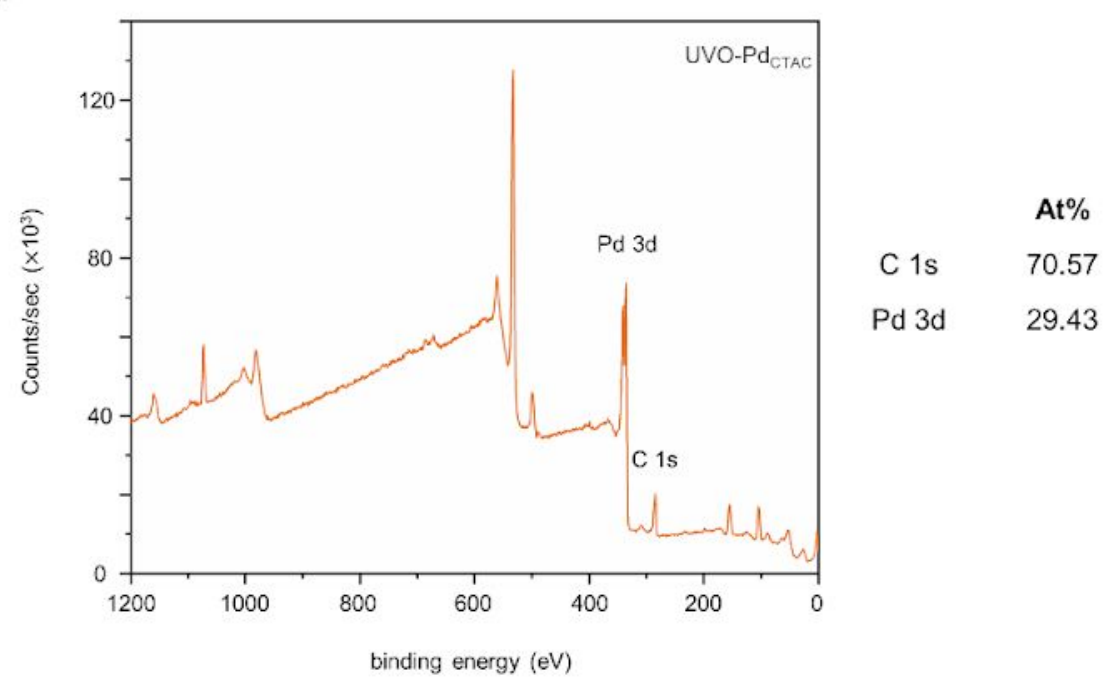

Figure S6. XPS survey scan analysis of (a) $\mathbf{P d}_{\text {CTAC }}$, and (b) UVO-Pd $\mathbf{d}_{\text {CTAC }}$ nanoparticles. The decreasing intensity of the $\mathrm{C}(1 \mathrm{~s})$ peak relative to the $\mathrm{Pd}(3 \mathrm{~d})$ peak after UVO treatment supports the loss of organic carbon surface ligands. The carbon C (1s) peak in the UVO treated samples is attributed to adventitious carbon. 
a

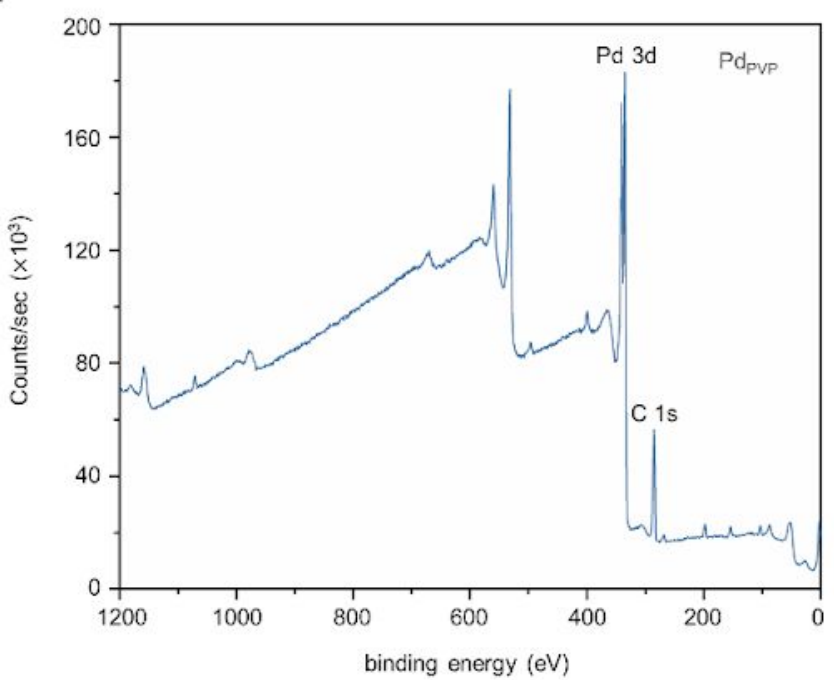

$\begin{array}{cc} & \text { At\% } \\ \text { C 1s } & 73.87 \\ \text { Pd 3d } & 23.76\end{array}$

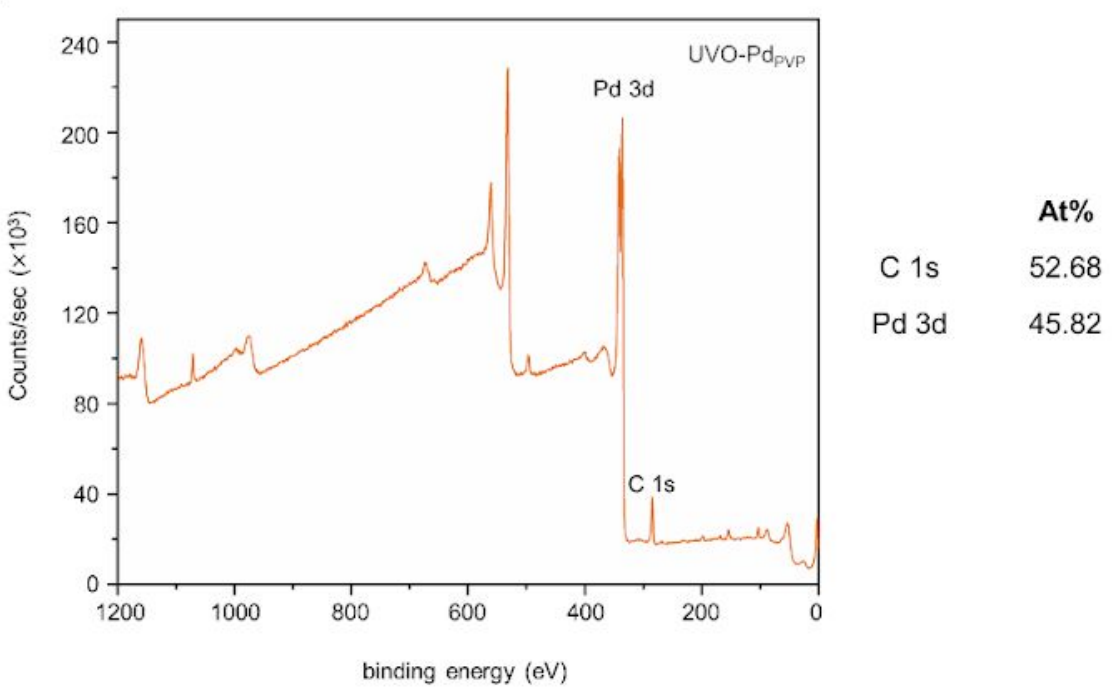

Figure S7. XPS survey scan analysis of (a) $\mathbf{P d}_{\mathbf{P V P}}$ and (b) UVO-Pd $\mathbf{P V P}_{\mathbf{P}}$ nanoparticles. The decreasing intensity of the $\mathrm{C}(1 \mathrm{~s})$ peak relative to the $\mathrm{Pd}(3 \mathrm{~d})$ peak after UVO treatment supports the loss of organic carbon surface ligands. The carbon C (1s) peak in the UVO treated samples is attributed to adventitious carbon. 


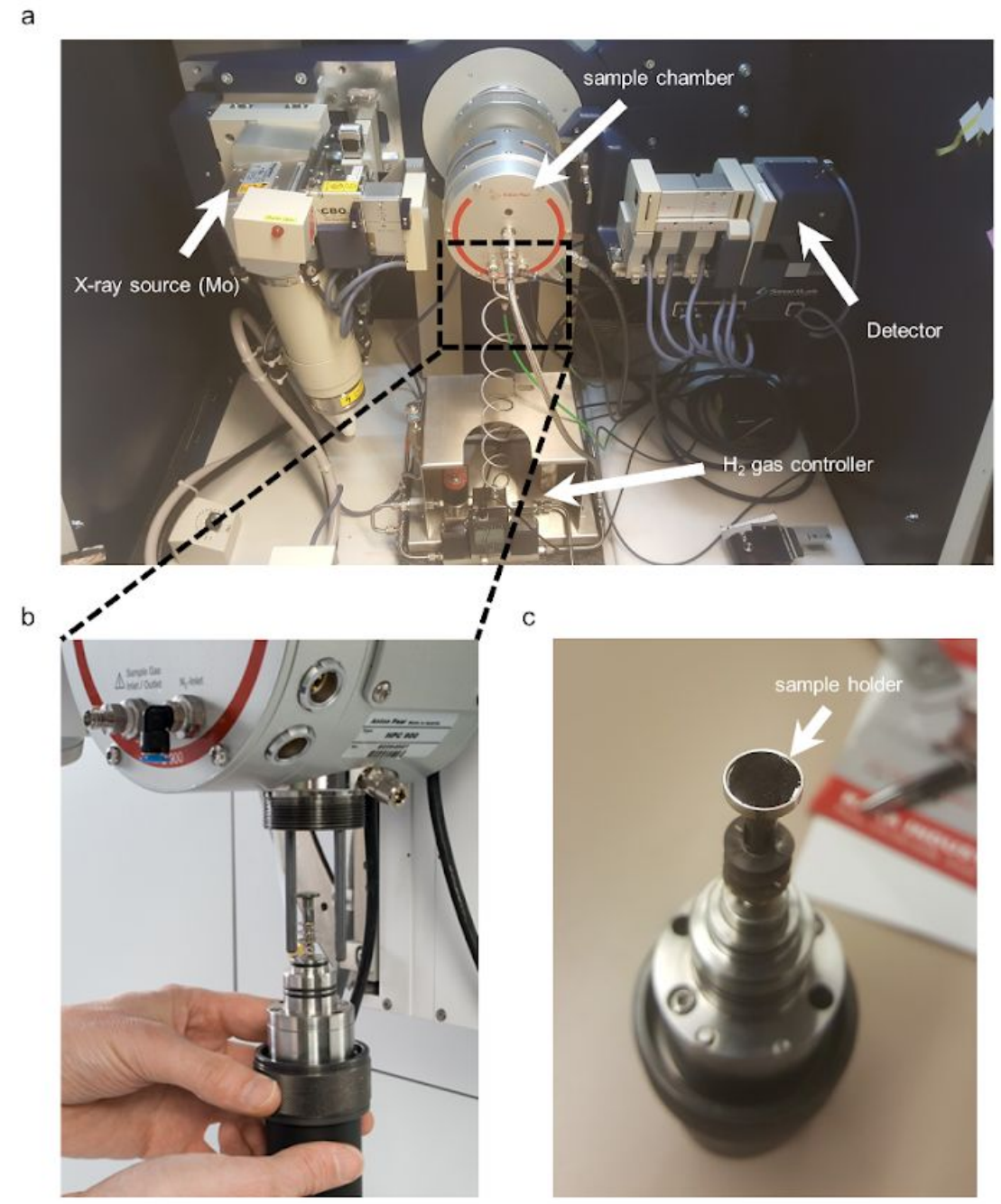

Figure S8. (a) Photograph of the in-situ XRD setup showing the Anton Parr HPC-900 sample chamber/pressure cell, X-ray source, hydrogen gas controller, and detector. (b) The sample stage and the sample mounting position on the pressure cell. (c) A dropcast $\mathbf{P d}_{\text {CTAC }}$ nanoparticle sample dried on the sample holder placed on the stage. 


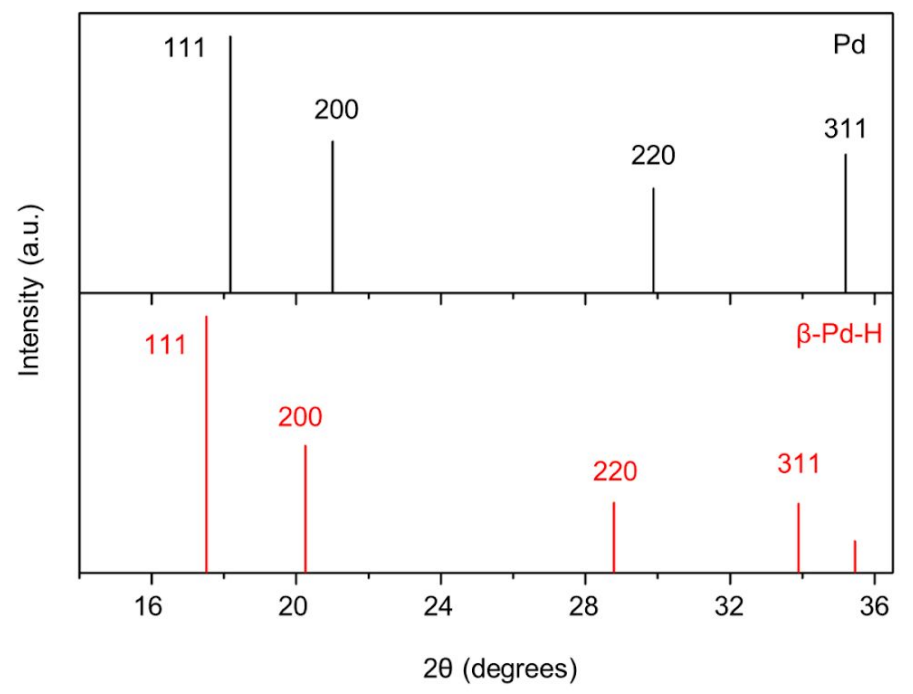

Figure S9. Standard XRD reference pattern of palladium (JCPDS: 00-046-043), and palladium hydride ( $\beta$-Pd-H) (JCPDS: 03-065-0557). $\beta-\mathrm{Pd}-\mathrm{H}$ peaks are shifted to smaller $2 \theta$ values compared to palladium because the palladium lattice expands upon hydrogen absorption. (X-ray source $=$ Mo; $\lambda=0.7107 \AA$.) 


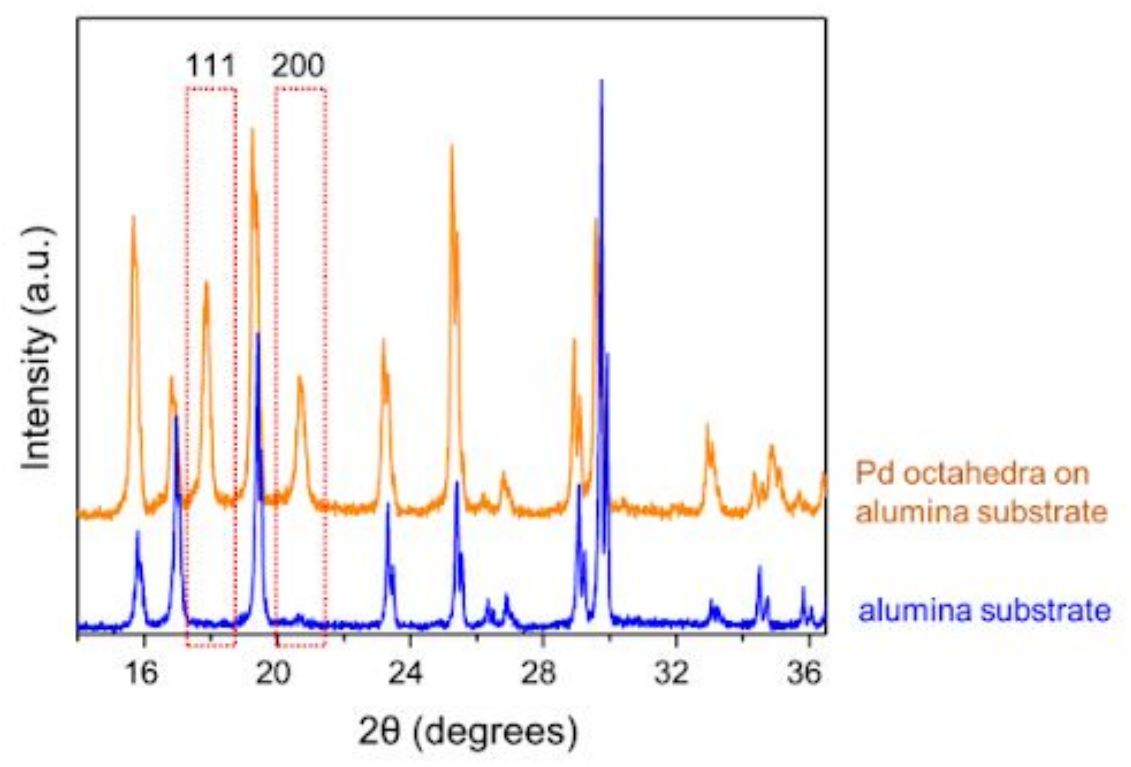

Figure S10. XRD pattern of $\mathbf{P d}_{\text {CTAC }}$ nanoparticles deposited on an alumina substrate (orange), and alumina substrate only without the nanoparticles (blue). The characteristic Pd (111) and (200) diffraction peaks do not overlap with the alumina substrate peaks. We chose to monitor the $\operatorname{Pd}(111)$ peak at $18.1^{\circ}(2 \theta)$ for the in-situ phase transformation studies. (X-ray source $=$ Mo; $\lambda=$ 0.7107 A.) 


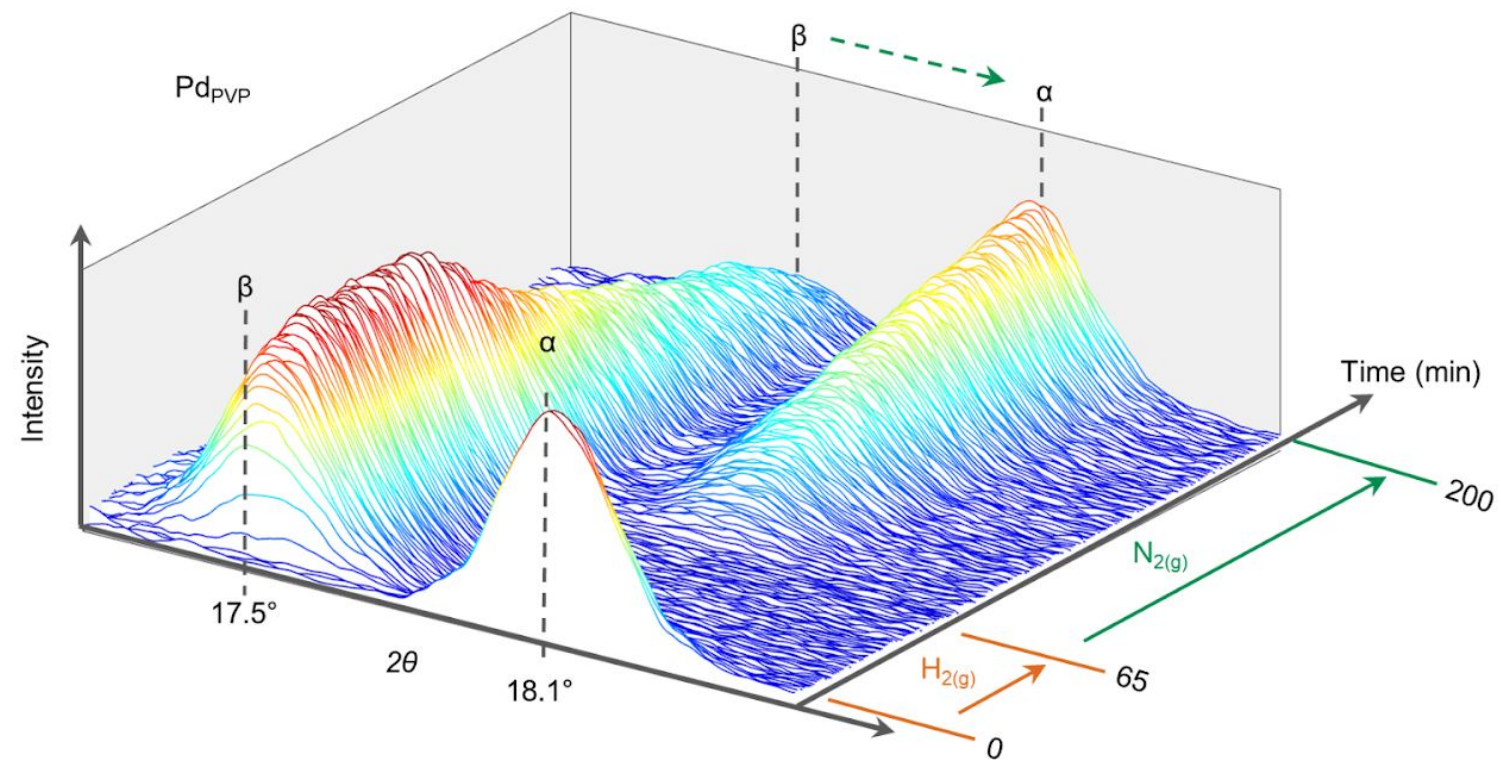

Figure S11. In-situ powder XRD diffraction wave plot of ligated $\mathbf{P d}_{\mathbf{P V P}}$ nanoparticles showing the hydrogen induced phase transformation as a function of time. Plot shows the successive short range scans between $16.5^{\circ}-18.6^{\circ}(2 \theta)$ at $\sim 1 \mathrm{~min} / \mathrm{scan}$. Introducing hydrogen $\left(5 \% \mathrm{H}_{2}: \mathrm{N}_{2}\right)$ results in the disappearance of the $\alpha$ phase $\left(18.1^{\circ}\right)$ and appearance of the $\beta$ phase $\left(17.5^{\circ}\right)$ peaks over time. Hydrogen desorption was initiated by replacing the $\mathrm{H}_{2}: \mathrm{N}_{2}$ atmosphere with pure nitrogen to affect a complete phase transition from the $\beta$ to $\alpha$ phase. $(\mathrm{X}$-ray source $=\mathrm{Mo} ; \lambda=0.7107 \AA$.) 


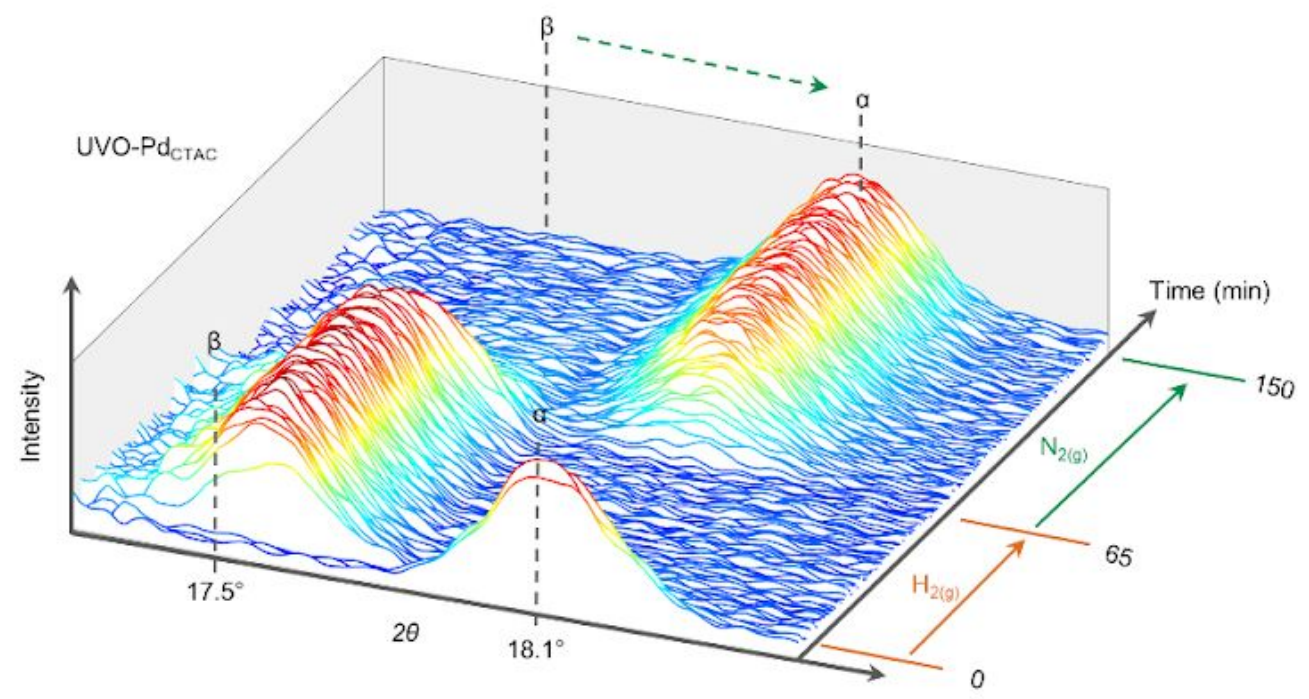

b

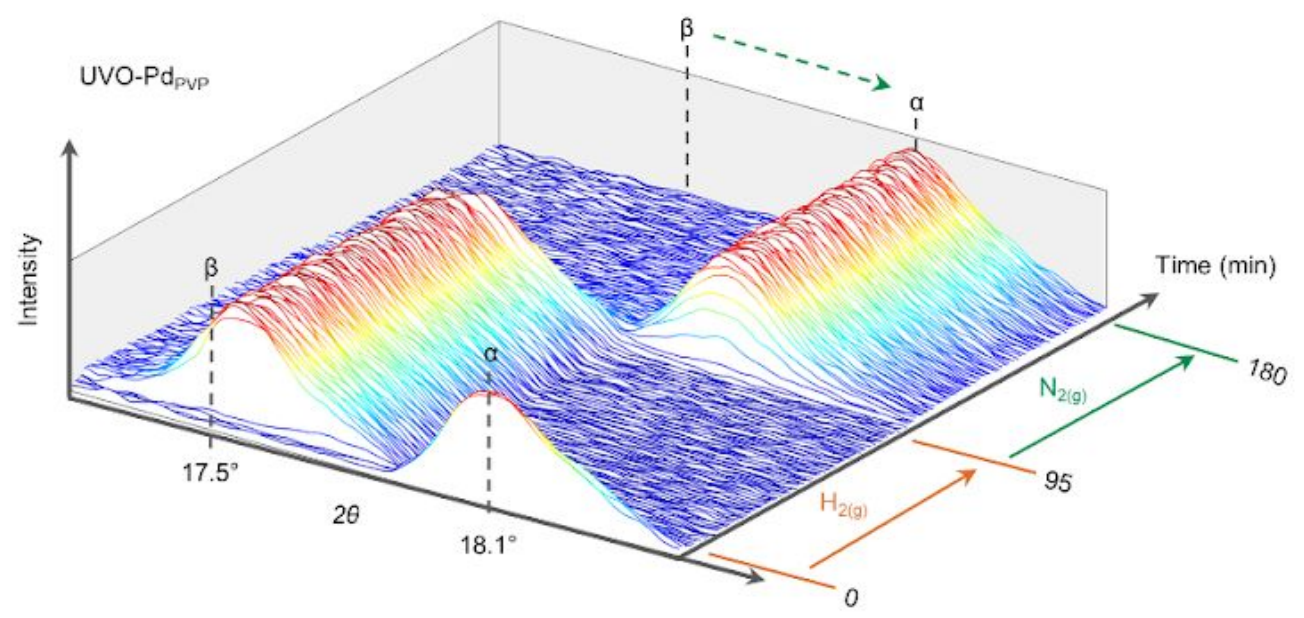

Figure S12. In-situ powder XRD diffraction wave plot of (a) UVO-Pd $\mathbf{C T A C}_{\text {and (b) UVO-Pd }}$ PVP nanoparticles showing the hydrogen induced phase transformation as a function of time. Plot shows the successive short range scans between $16.5^{\circ}-18.6^{\circ}(2 \theta)$ at $\sim 1 \mathrm{~min} / \mathrm{scan}$. Introducing hydrogen $\left(5 \% \mathrm{H}_{2}: \mathrm{N}_{2}\right)$ results in the disappearance of the $\alpha$ phase $\left(18.1^{\circ}\right)$ and appearance of the $\beta$ phase $\left(17.5^{\circ}\right)$ peaks over time. Hydrogen desorption was initiated by replacing the $\mathrm{H}_{2}: \mathrm{N}_{2}$ atmosphere with pure nitrogen to affect a complete phase transition from $\beta$ to $\alpha$ phase. (X-ray source $=$ Mo; $\lambda=0.7107 \AA$.) 
a

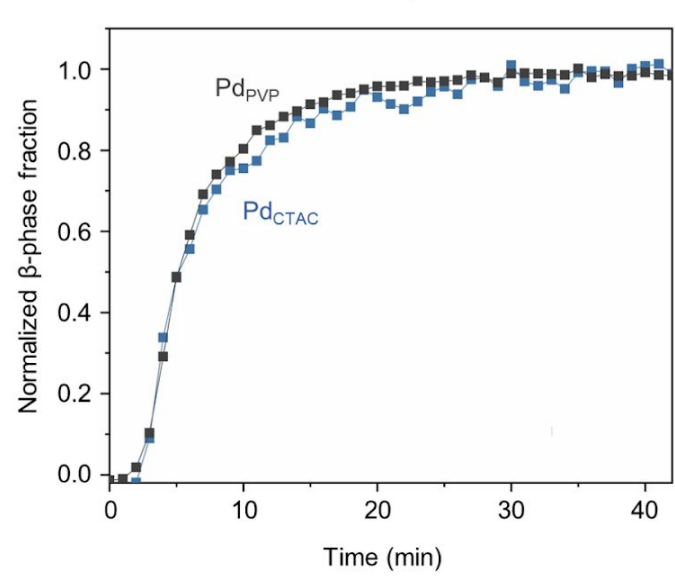

b H-desorption

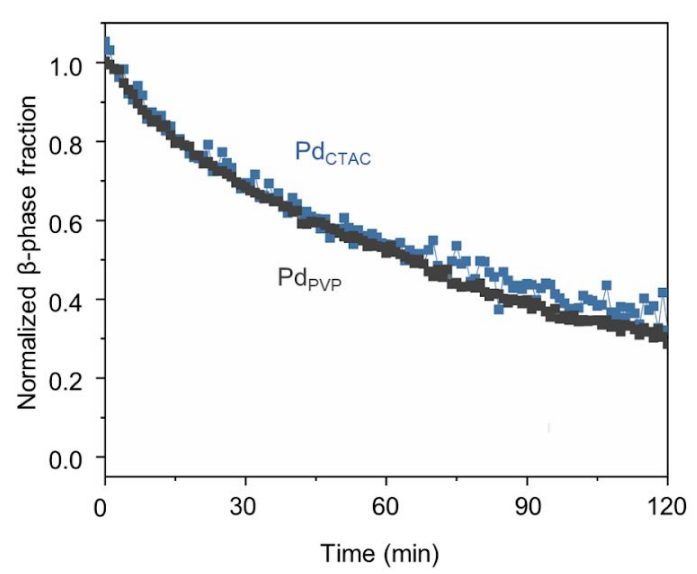

Figure S13. Normalized $\beta$-phase fraction during (a) hydrogen absorption and (b) hydrogen desorption as a function of time for ligated octahedral $\mathbf{P d} \mathbf{d}_{\mathrm{CTAC}}$ and $\mathbf{P d}_{\mathrm{PVP}}$ nanoparticles. 